\title{
Las redes sociales y la cultura de la autopromoción. Apuntes para una teoría de la identidad mosaico
}

\section{Social Networking Sites and Self-Promotional Culture. Notes for a Theory of the Mosaic Identity}

\author{
Lucía Caro Castaño. Universidad de Cádiz (lucia.caro@uca.es)
}

Recibido: 8/3/2016 - Aceptado: 22/12/2016

\section{Resumen:}

Este trabajo exploratorio teoriza acerca del modo en que las redes sociales digitales favorecen, en tanto que tecnologías de la identidad, un modo de concebir y presentar la identidad individual en términos de autopromoción. Como resultado de la normalización de esta lógica -coherente con la cultura promocional del capitalismo tardío-, crece la incorporación de prácticas como la marca personal en la comunicación cotidiana de los sujetos, la percepción del perfil social como un micromedio de comunicación y la concepción de la red de contactos a modo de audiencia personal. En síntesis, se señalan cuatro tendencias centrales en la concepción y presentación de la identidad que favorecen estos servicios web: una concepción distribuida y fragmentaria del yo, donde las teselas de los massmedia se convierten en contenidos clave para expresar la subjetividad; una tendencia a la cuantificación de las relaciones y los afectos; la percepción de encontrarse en una ineludible competencia con los otros; y la normalización de la presentación audiovisual del yo como un material comunicativo capaz de atraer la atención y comunicar autenticidad.

\section{Palabras clave:}

Identidad; redes sociales; autopromoción; publicidad; cultura mosaico.

\begin{abstract}
:
This exploratory work theorizes about how social networking sites favor, as identity technologies, a way of conceiving and presenting individual identity in self-promotional terms. As a result of the normalization of this logic-which is coherent with the late capitalism' promotional culture-, it is growing the incorporation of self-branding practices in the users's daily communication. Besides, it is increasing the perception of the social profiles as micro-media and the interpretation of the own network as a personal audience. In brief, four main trends in the presentation of identity which are promoted by these web services are identified: a distributed and fragmented conception of the self, where the tiles from mass media become key content to express subjectivity; a tendency to quantify relationships and affections; the perception of being in an unavoidable competition with others; and standardization of the audiovisual presentation of self as a communicative material capable of attracting attention and communicate authenticity.
\end{abstract}

\section{Key words:}

Identity; social networking sites; self-promotion; advertising; mosaic culture. 


\section{Introducción}

Las demandas de la Segunda Modernidad acerca de la identidad como proyecto reflexivo (Giddens, 1994; Sibilia, 2008; Cover, 2014) encuentran en las redes sociales digitales un formidable vehículo para que el individuo pueda definirse ante los otros a través de sus elecciones culturales y de consumo (Liu, 2007; Rendueles, 2013a, 2013b). Estas plataformas han sido estudiadas como tecnologías de la subjetividad (Marwick, 2013) o de la identidad (Cover, 2014), herederas ambas formulaciones de la propuesta de Foucault (1990) sobre las tecnologías del yo: tecnologías de acceso y construcción de la subjetividad, que permitirían el cuidado de uno mismo.

Sin embargo, en el actual estadio del capitalismo, donde el mercado se convierte en el principio organizador de la vida social (Wernick, 1994; Brown, 2003; Verdú, 2007; Rendueles, 2013a), las plataformas de la web 2.0 constituyen "tecnologías neoliberales de la subjetividad que enseñan a los usuarios cómo triunfar" (Marwick, 2013: 14), logrando así que el autogobierno del individuo converja con los principios del sistema. Así, como se expondrá más adelante, la cultura de estos espacios y diversas decisiones en torno al diseño de interacción contribuyen a promover la libre adopción de una serie de valores y prácticas que permiten trasladar el proceso de acumulación capitalista a la experiencia de la vida cotidiana en relación con los afectos, las vinculaciones y la concepción de la propia identidad.

En este sentido, el presente trabajo reúne bajo la metáfora de la identidad mosaico ${ }^{1}$, una serie de tendencias que pueden observarse en la comunicación de los usuarios de acuerdo con la actual estabilización de estos servicios web² . En concreto, se analizan cuatro tendencias centrales en la concepción y presentación de la identidad que favorecerían estas plataformas: una concepción distribuida y fragmentaria del yo, donde las teselas de los massmedia se convierten en contenidos clave para expresar la subjetividad; una tendencia a la cuantificación y acumulación de las relaciones y los afectos; la percepción de encontrarse en una ineludible competencia con los otros por la visibilidad; y la creciente presentación audiovisual del yo como un material comunicativo capaz de atraer la atención y trasladar autenticidad y veracidad.

\section{Estado de la cuestión}

Los actuales trabajos académicos acerca de las implicaciones de las plataformas de redes sociales digitales en la concepción de la identidad (Zhao, Grasmuck y Martin, 2008; Cáceres, Ruiz-San Román y Brändle, 2009; Andrejevic, 2010; Arcila, 2011; Arda, 2011; Davis, 2012; Harper, Whitworth y Page, 2012; Vivienne, 2012; Caro Castaño, 2012; Zappavigna, 2012; Marwick, 2013; Serrano-Puche, 2013; Van Dijck, 2013; Zajc, 2013), pueden enmarcarse en la tradición de aquellas

1 La noción de identidad mosaico ya ha sido objeto de una primera aproximación (Caro Castaño, 2012) si bien el concepto se reformula en este trabajo como teoría.

2 El diseño de este tipo de servicios se concibe como dependiente de las prácticas que desarrollen los usuarios, en una eterna "fase beta", siempre abierta a modificaciones, empleando la terminología de ingeniería de software.

14 | n² 24, pp. 13-36 | doxa.comunicación 
investigaciones que analizan cómo las tecnologías de la comunicación median la imaginación en la construcción de subjetividad (Haraway, 2006; Gergen, 2010; Kember y Zilinska, 2012; Nakamura, 2014).

Siguiendo la que probablemente sea la definición más extendida en la academia sobre estas plataformas, las redes sociales digitales constituyen redes egocéntricas (Boyd y Ellison, 2008), es decir, la red se construye a partir de cada sujeto -egofrente a construcciones precedentes como las comunidades virtuales, basadas en un interés común en torno a un tema específico (Kozinets, 2010). Asimismo, estos servicios web no sólo elaboran la idea de lo social desde el sujeto individual (Rendueles, 2013a, 2013b; Zajc, 2013), sino que potencian la presentación de un individuo no-anónimo (Zhao, Grasmuck y Martin, 2008), pues la plataforma interpela constantemente a los usuarios para que aporten datos sobre su vida presencial (lugar de trabajo, geolocalización de las publicaciones, teléfono, etc.).

Todo ello facilita la incorporación a estas plataformas de vínculos preexistentes en el mundo presencial (Ellison, Steinfield y Lampe, 2007; Zhao, Grasmuck y Martin, 2008; Hampton et al., 2011). De este modo, identidad legal y virtual tienden a converger en estos espacios, llegando a detectarse, por ejemplo, entre los jóvenes españoles, la interpretación del anonimato en la red como un hecho moralmente reprobable (Cáceres, Ruiz-San Román y Brändle, 2009). Así, frente a la experimentación con las máscaras que permitieron las plataformas previas a la web 2.0 (Arda, 2011) -en mundos virtuales, foros, canales de chat, etc.-, los sujetos tienden a concebir su presencia en las redes sociales como una prolongación de su realidad cotidiana, donde los planos presencial y no presencial convergen en una realidad única (Jurgenson, 2012; Reig, 2012; Pérez Subías, 2012): lo que sucede en Facebook no queda circunscrito a Facebook, como sí puede suceder en entornos como los mundos virtuales.

Una de las consecuencias directas de esta fusión es que las estrategias desarrolladas por los usuarios en estas plataformas buscan producir efectos en el mundo presencial (Lampel y Bhalla, 2007; Kozinets, 2010; Davis, 2012; Woermann, 2012). Ello explicaría las inversiones de tiempo y esfuerzo que desarrollan muchos usuarios para ampliar su número de contactos y para la creación y difusión de contenidos propios gratuitos que le permitan hacerse más visible, obtener autoridad o definir su posicionamiento en línea (Myers, 2010) -en términos de brandingy de visibilidad en buscadores-. Así, en el marco de una cultura promocional, donde la subjetividad deseable es la de un "sujeto-marca" (Rowan, 2012) capaz de venderse a sí mismo, las redes sociales serían observadas como micromedios de comunicación (Del Fresno, 2012) que permitirían construir audiencias conectadas (Marwick y Boyd, 2011; Marwick, 2013) que den acceso a la visibilidad masiva.

Finalmente, cabe señalar que el análisis de las redes sociales como medios integrados en el dispositivo de poder neoliberal, capaz de hacer-hacer, siguiendo la terminología foucaltiana, ha sido planteado por diversos autores (Han, 2013; Marwick, 2013; Rendueles, 2013a; Colombo, 2013), mientras que otros investigadores han insistido en la creciente concepción instrumental que favorecen estas plataformas de los afectos (Illouz, 2007), las habilidades comunicativas (Fuchs y Sevignani, 2013) y la propia identidad como producto de consumo (Hearn, 2008; Deresiewicz, 2011; Page, 2012). 


\section{Objetivo y pregunta de investigación}

Las plataformas de redes sociales son observadas en este trabajo como constructos sociotécnicos, esto es, su análisis debe considerar tanto sus infraestructuras materiales como los significados sociales, los imaginarios y las metáforas que circulan y contribuyen a dar sentido a las prácticas de los usuarios. Por tanto, estos espacios no deben entenderse exclusivamente como artefactos tecnológicos; muy al contrario, deben analizarse considerando las relaciones de interdependencia que se producen en estos nuevos lugares sociales, así como las sinergias que nacen entre tecnologías, discursos y prácticas. De este modo, si bien la herramienta plantea una serie de potencialidades comunicativas y de expresión de la identidad, la agencia es compartida entre el individuo y sus herramientas (Lasén, 2009, 2012; Kember y Zylinska, 2012). Así, son los usos socialmente situados que hacen las personas y las comunidades de estas potencialidades los que codeterminarán su evolución.

Partiendo de este planteamiento, se aborda en este trabajo el siguiente objetivo: la definición de una teoría en torno a la forma concreta de concebir la subjetividad y presentar la identidad social en las plataformas de redes sociales digitales. En este sentido, se busca comprender cómo interactúan los sujetos con el medio, las potencialidades y limitaciones que plantean las diferentes plataformas para la interacción y la proyección del yo, y el modo en que estos diseños favorecen la preeminencia de unos discursos en sus prácticas frente a todos los posibles.

Para alcanzar el objetivo de investigación expuesto, se formuló la siguiente pregunta: ¿Qué tipo de subjetividad contribuyen a producir las redes sociales digitales de acuerdo con las potencialidades de estos artefactos tecnológicos?

\section{Metodología}

Este trabajo tiene un carácter exploratorio y se centra en un metaanálisis bibliográfico que permite estudiar las tendencias y transformaciones en los modos de concebir y presentar la identidad y la sociabilidad derivada de ésta en las redes sociales digitales. Estas plataformas web son analizadas en tanto que constructos sociotécnicos, desde la perspectiva del enfoque CTS (Ciencia, Tecnología y Sociedad) (Pinch y Bijker, 1984). Dado el carácter teórico de este trabajo, las conclusiones alcanzadas en esta investigación habrán de ser puestas a prueba en futuros trabajo empíricos.

Por otra parte, y como vía de acceso directo al objeto de estudio, la revisión bibliográfica es complementada -aunque con un peso secundario en el diseño de investigación- con la utilización de la observación etnográfica digital (Hine, 2004; Kozinets, 2010; Ardèvol y Gómez-Cruz, 2012; Del Fresno, 2012) en diferentes plataformas de redes sociales. 


\section{Mediatización y cultura mosaico}

La elección de la metáfora del mosaico al nombrar esta propuesta teórica busca comunicar del modo más directo posible una serie de ideas que se consideran clave en este trabajo en relación con el modo en que se presenta la identidad en estas plataformas. Por una parte, se apela a la noción más popular del término, tanto en su significado denotativo -como un conjunto compuesto por elementos de tamaño, color, procedencia y/o textura diferentes que conforman una obra-, como en sus significados metafóricos -como sinónimo de diversidad cultural y/o étnica, por ejemplo-.

Así, se busca emplear la imagen del mosaico para retratar el modo en que estos espacios se basan en la construcción de una estructura de red donde las vinculaciones definen el lugar que ocupa el sujeto en el conjunto social, más centrada o más cercana a los márgenes -con mayor o menor capacidad de influencia en la red, respectivamente-. Se emplea además la imagen de las teselas del mosaico, ya que la identidad individual se tangibiliza en las redes sociales mediante un caudal de ítems de información de procedencia y naturaleza diversa (creaciones propias, comentarios de amigos, de desconocidos; fragmentos de películas, programas de televisión, noticias, etc.) que contribuyen a construir la narrativa del yo.

Por otra parte, y ya en relación con la literatura académica en comunicación, se busca hacer un reconocimiento expreso a algunas de las aportaciones de Moles (1978) y su propuesta sobre la "cultura mosaico" que, de acuerdo con su teoría de la sociodinámica de la cultura, habría sido generada por los medios de comunicación de masas, siendo esta cultura una de las mediaciones que se consideran centrales para comprender el modo de concebir la identidad en estos espacios. No obstante, como se verá en las próximas páginas, se encuentran salvedades notables en relación con la visión mecanicista y unidireccional de la propuesta de Moles, donde la agencia es exclusivamente atribuida a los massmedia y las élites gobernantes.

La apelación a la cultura mosaico, por tanto, avanza uno de los útiles teóricos centrales de este trabajo, como es la noción de mediatización. El término hace referencia a la cualidad de omnipresencia de los medios de comunicación de masas y a su capacidad de afección sobre el modo en que los individuos aprehenden la realidad en múltiples niveles (Silverstone, 2002; Martín Barbero, 2002, 2008; Sampedro, 2004; Krotz, 2007; Couldry, 2008, 2014; Hjarvard, 2013).

Asimismo, la mediatización ha sido empleada para insistir en la transformación que los massmedia han operado en diversos ámbitos sociales al adaptarse éstos a la representación mediática que los media han construido en torno a ellos, desde la comunicación política al modo en que se concibe cómo debe ser una boda en la actualidad (Bird, 2010). En relación con los medios sociales, Smith y Watson (2014) hablan de "automedialidad" para hacer referencia al modo en que las características de estos medios afectan a la presentación de la identidad en ellos (2014: 77), insistiendo en que la estética propia de la automedialidad es la del "collage, mosaico, pastiche" (ibídem: 78), contribuyendo así a la ruptura con el mito de la concepción esencialista de la identidad (Mischel y Morf, 2005; Haraway, 2006; Gergen, 2009, 2010). 


\subsection{Aportaciones de la cultura mosaico}

Para Moles, la lógica de los medios de comunicación masivos y su preponderancia en la configuración de la cosmovisión social provocó una ruptura de los nexos causales y racionales propios del modelo humanista racional, potenciando la lógica fragmentaria entre los contenidos mentales. De acuerdo con el autor francés, lo importante no era la pérdida de una relación de conexión lógica entre contenidos concretos yuxtapuestos, sino el efecto acumulativo que esta práctica tendría en los individuos: la anulación de la capacidad de producir interpretaciones causales globales de la realidad. Dentro de la cultura mosaico, los fragmentos se ensamblan sin seguir criterio alguno, de modo aleatorio y por yuxtaposición, de acuerdo con Moles.

El sociólogo propuso el concepto de "culturema" para referirse a las unidades de contenido que permiten a los sujetos comprender los sistemas culturales, los útiles para entender la cultura que son seleccionados por los media sin tomar en cuenta su trascendencia social (1978: 302). Esta falta de orden, generaría una amalgama de temas que, aún incrementando su difusión, no habrían producido un mayor número de asociaciones entre culturemas, es decir, no habrían alumbrado ideas nuevas, sino más bien un fenómeno de revival (ibídem: 308). De este modo, que la mayoría de los culturemas provenga de los massmedia devendría, según Moles, en una banalización de los contenidos y en la desaparición de herramientas que permitan a los sujetos desentrañar la urdimbre de los discursos interesados que convergen en la construcción mediática de la cotidianidad.

Sin embargo, las posibilidades de expresión, manipulación y difusión que las plataformas de autocomunicación de masas ofrecen hoy a los individuos han puesto de manifiesto que las audiencias son activas en su procesamiento de la información que reciben de los media, y que son capaces de producir su propio contradiscurso hacia aquellos sesgos informativos que no coinciden con su punto de vista sobre la realidad. Así, si se realiza una equiparación de los culturemas enunciados por Moles con los memes políticos que son creados y remezclados por los usuarios en los medios sociales, es evidente la existencia de una constante manipulación por parte de los miembros de las audiencias sobre los productos massmediáticos para pervertir su significado original (Jenkins, 2008; Rodríguez, 2013; Shifman, 2014).

\subsubsection{La narración fragmentaria como síntoma de espontaneidad}

Pese a que se encuentran necesarias matizaciones como la precedente para la recuperación de la propuesta de Moles, la identidad mosaico que se presenta en este trabajo se relaciona directamente con el modelo cultural enunciado por el sociólogo y retoma dos de sus rasgos. El primero es el carácter fragmentario del discurso del yo, es decir, la inexistencia de una narración lineal en términos de autoría, formato o temática. Dado que muchos de estos contenidos no serán generados por el usuario, es el exotismo en la selección de las teselas lo que comunica una singularidad que, aun siendo secundaria, es suficiente: lo importante es el criterio individual de consumo que se expresa a través de estas elecciones. 
Una idea que conecta con el discurso de los estilos de vida y la búsqueda de seguridad ontológica contemporánea (Giddens, 1994), y que puede relacionarse con la propuesta del "yo extendido" de Belk (1988): aquello de lo que el sujeto elige rodearse expresa una subjetividad única, un proyecto de vida, un punto de vista y de acción específicos sobre la realidad.

Se considera además que la narración del yo no debe mantener una tematización férrea en sus publicaciones dentro de estos espacios, ya que cuando una persona emplea su perfil para compartir exclusivamente informaciones relativas a su disciplina profesional o a la temática en la que se posiciona como experto/a, tenderá a ser percibido/a como una persona inauténtica, una especie de autómata, al restringir completamente el acceso de los otros a informaciones más personales e íntimas. Así, dentro del proceso iniciado por los massmedia, se alcanza una nueva etapa en la que se normaliza la exposición de contenidos otrora propios de los espacios privados e íntimos (McLuhan, 1980; Meyrowitz, 1985; Thompson, 2001, 2010; Sibilia, 2008; Han, 2013), en paralelo a un gran proceso social en el que la separación personal/profesional que se fraguó en la sociedad industrial tiende a volver a fundirse.

Entre los factores que contribuyen a esta creciente fusión, cabe destacar el fenómeno de la copresencia, que ha contribuido a generar lo que Thompson denominó la "sociedad de la autorrevelación”: “los líderes políticos ya pueden dirigirse a sus súbditos como si lo hicieran ante familiares y amigos” (2001: 66). Y, al igual que lo han venido haciendo los líderes políticos, las celebridades y, en general, cualquier figura pública, ahora cualquier individuo que observe sus perfiles sociales como micromedios de comunicación puede también dirigirse a una multitud. Por otra parte, y en relación con el tipo de vivencia que ofrecen estos medios, es interesante destacar cómo la primacía de la experiencia personalizada que ofrecen los algoritmos de las redes sociales y los buscadores han transformado la red en una esfera íntima: “[e]sta cercanía digital presenta al participante tan solo aquellas secciones del mundo que le gustan. Así se desintegra la esfera pública, la conciencia pública, crítica, y privatiza el mundo" (Han, 2013: 69). Eliminado lo público del tablero, queda la "publicación de la persona", de acuerdo con Han: el escaparate y el mercado frente al espacio social en común.

\subsubsection{Los contenidos mediáticos como elementos centrales de la identidad}

Otro de los rasgos que se retoman de la cultura mosaico es el origen mediático de la mayoría de los contenidos de las teselas compartidas por los usuarios de las redes sociales, al considerarse a las industrias culturales y a los massmedia como configuradores del imaginario colectivo, como señalaba Moles (1978):

"La prensa, la radio, los discos de éxitos, las reproducciones de pinturas [...] ejercen un peso abrumador sobre el conjunto del sistema social, rigen nuestra cultura al filtrarla, extraen de ella elementos particulares y les otorgan importancia, valorizan algunas ideas y devalúan otras y polarizan completamente el campo cultural” (ibídem: 100-101). 
La urdimbre de los contenidos mediáticos en la vida cotidiana se hace patente en las redes sociales. Ello puede observarse no sólo en la ingente cantidad de contenidos producidos por los media que los usuarios comparten, sino, especialmente, en la proliferación de aplicaciones que proponen al usuario definirse ante los demás de acuerdo con distintos productos de las industrias culturales. Así, por ejemplo, existen multitud de tests en Facebook cuyas respuestas acaban clasificando al entrevistado de acuerdo con personajes de películas, series, etc: “¿En qué película se basa tu vida?”, “¿Qué personaje de Star Wars eres?”, etc. Este tipo de aplicación enfatiza la idea de que la subjetividad puede desgranarse en elecciones culturales y de consumo, materiales culturales mainstream producidos por las industrias culturales que ayudarían al sujeto a expresar ante los demás su pertenencia a un determinado grupo o subgrupo cultural.

\section{Rasgos de la identidad mosaico}

La ubicuidad de las redes sociales y su integración en la vida cotidiana han contribuido a la emergencia de un nuevo sentir del mundo que modifica el del propio cuerpo y el contexto, donde crece el peso de la dimensión estratégica en la interacción social que planteó Goffman en su clásica teoría de la interacción dramatúrgica (1967, 1969, 1991, 2006). A continuación se revisan los rasgos principales observados en torno al modo específico de ser y estar en el mundo que proponen las redes sociales.

\subsection{Un yo co-construido, fragmentario y fluctuante}

El diseño de la interacción y el modo en el que se muestra el perfil y los vínculos del usuario en las redes sociales digitales plantea una estructura que potencia una concepción distribuida de la identidad social, dado que estas plataformas permiten al sujeto manipular la representación de su grafo social. Así, como ha señalado Zappavigna (2012: 2), "la red social consiste en el uso de Internet para representar relaciones más que para compartir información, aunque las dos funciones están claramente interconectadas". La herramienta ofrece al sujeto, como hacía el reflejo del agua calma para Narciso según McLuhan (1980), observarse de un modo nuevo: un yo distribuido, en continua co-creación y negociación con el grupo primario y con la red extensa. En definitiva, las redes sociales permiten al individuo tangibilizar, traducir y renegociar su ubicación en el grafo social digital, con personas, organizaciones, creaciones culturales, ideológicas, etc.

Siendo la función central la expresión de las vinculaciones, no resulta extraño que las aplicaciones más virales en una red social generalista como Facebook sean aquellas que se centran en el sujeto y en la representación de sus vínculos y biografía, como la aplicación de vídeo que creó Facebook en 2014 para celebrar sus 10 años de existencia o, más recientemente, la $a p p$ para crear un vídeo en el que el usuario puede hacer un clip de un minuto de duración dedicado a un amigo. Esta pieza sigue siempre un mismo esquema y narrativa estándar, de manera que lo que cambia de un vídeo a otro es la selección de las publicaciones concretas con las que se quiere comunicar al sujeto en cuestión y al resto de sus contactos el valor 
especial de su relación de amistad. Se trata, por tanto, de un tipo de vídeo prefabricado que permite al usuario expresar ante los otros cuáles son los vínculos preferenciales dentro de su red.

Traducir la mismidad y reconstruir el lugar-en-el-mundo en el plano online permite diferentes grados de libertad al sujeto en la fidelidad de esta reproducción de su vida presencial que será, en cualquier caso, performativa para ambos planos de realidad -presencial y no presencial-. No obstante, como ya se ha señalado, el aumento del trasvase de relaciones de la realidad presencial a la no presencial, reduciría estas posibilidades creativas en la definición de una identidad digital estable y conectada.

\subsubsection{La web dialéctica y el interaccionismo simbólico}

Dado el carácter dialéctico de la web (Senft, 2008; Deuze, 2012), estos espacios no sólo permiten que el sujeto maneje de modo estratégico la presentación de su identidad y sus vinculaciones, sino que hace posible que los otros participen de esta creación. La identidad en estos espacios es co-construida materialmente, realizando así de un modo físico -la materialidad de los bits- la proposición central del interaccionismo simbólico (Blumer, 1980; Mead, 2009) y de la teoría del reconocimiento de Honneth (1998). Así, el modo en que el otro se relaciona en público con el sujeto, responde o calla ante las publicaciones de este, se fotografía o no con él, etc. informa al sujeto acerca de la consideración de los otros hacia él -especialmente de los vínculos débiles-, al mismo tiempo que funciona frente a las audiencias como un "principio de garantía" (Walther y Parks, 2002) de aquello que el sujeto expresa ser y del lugar social que pretende trasladar en las plataformas online.

Además de la posibilidad de traducir y, en ocasiones, mejorar la posición en el grafo social, las redes sociales digitales son empleadas por los usuarios para el mantenimiento de los vínculos en una red social en constante crecimiento. Como indica Miller (2008), las redes sociales serían "medios fácticos" (2008: 395), cuya función primordial es la de permitir interacciones comunicativas que sostengan la vinculación en el tiempo con los otros, conexiones especialmente costosas de mantener cuando se trata de vínculos débiles. Ello explicaría el escaso valor informativo de gran parte de los contenidos que los usuarios ponen en circulación, ya que lo central no es lo que fluye por los vínculos, sino que la circulación de esos ítems refuerza la sensación de copresencia. En definitiva, la publicación de estas informaciones, aunque de escaso interés y/o elaboración, ayudaría a mantener los vínculos sociales en el tiempo con una menor dedicación de recursos personales.

Por otra parte, y como ya se ha indicado en relación con la teoría de la sociodinámica de la cultura de Moles (1978), el caudal de actualizaciones que mantienen los usuarios sigue una lógica fragmentaria, en cuanto a la procedencia y autoría de estas teselas de contenido, y a la lógica narrativa que desarrolla cada sujeto que, si bien puede ser completamente distinta en cada individuo, tiende a presentar una lógica de flujo de fragmentos, crecientemente breve. Esta tendencia a la breve- 
dad -fomentada especialmente por Twitter y sus 140 caracteres- ha sido enfatizada con la incorporación de plataformas como Vine, los vídeos cortos que permite Instagram o la instantaneidad de la narrativa del gif que amplía las capacidades expresivas de los usuarios en estos espacios.

\subsection{Personificación, reificación y cuantificación del yo}

En la actual fase del capitalismo ficción ${ }^{3}$, las relaciones entre objeto y sujeto han alcanzado un nuevo estatuto de la existencia, según Verdú: el "sobjeto": transformándose los objetos en “[s]eres animados que reciben vida a través de la acción entre ellos y con los sujetos” (2007: 103). Esta nueva dialéctica, deriva según el sociólogo español en un fenómeno animista (ibídem: 107) en el que cualquier objeto o realidad -las ciudades, por ejemplo, ahora "inteligentes" (Mitchell, 2007) o "espacios sensibles" (Di Siena, 2011)- es presentado hoy con atributos de lo humano.

La dialéctica descrita por Verdú se centra en la idea de que los productos en la fase del capitalismo de ficción han sido dotados de atributos sensibles, en un proceso que ha incorporado a la apariencia (visión), el trabajo sobre las texturas y acabado (tacto), la simbología de marca (intelecto), el sonido -el eslogan sonoro de una puerta que se cierra en Volkswagen, el sonido atemperado del teclado de un Mac, etc.- y el olor (ibídem: 108) propio del marketing sensorial. Desde la perspectiva de este trabajo, este proceso animista se completa de un modo más profundo en el entorno digital, en tanto que ambos, sujetos y objetos, son traducidos a un mismo material y espacio.

Es importante destacar en este punto, una de las ideas indicadas por Verdú en relación con este proceso en el que las marcas se convierten en herramientas esenciales para la expresión de la seguridad ontológica: la percepción del sujeto no es la de sentirse cosificado; muy al contrario, siente que los objetos, las marcas, les "personifican" (ibídem: 113). El paso al ámbito digital ayudará a profundizar aún más un proceso de equiparación ontológica entre personas y marcas, una transformación que contribuye a explicar la facilidad y alegría con la que muchos individuos se han entregado a la tarea de convertirse en una marca personal, o el hecho de que los consumidores más jóvenes puedan interpretar su vinculación con sus marcas favoritas como algo más que una relación de preferencia hacia una opción de consumo: como una vinculación afectiva (VVAA, 2014) e identitaria.

La tendencia a igualar el estatuto de la existencia del ser humano y el objeto se propone al consumidor como un discurso de personificación y no de cosificación. Siguiendo con este razonamiento, gestionar la identidad social como una marca personal no sería percibido por el individuo como un proceso que devalúa lo humano en su reificación ${ }^{4}$, sino como un tra-

3 Verdú denomina capitalismo ficción a la etapa posterior a la del capitalismo de consumo, superada en gran medida por el descrédito de la publicidad, según el autor (2007: 110). Se trata de una fase en la que el capitalismo habría iniciado el proyecto de sustituir lo real por lo producido.

4 La noción de reificación se emplea aquí en un sentido no marxista, siguiendo a Honneth (1998) e Illouz (2007). Se denomina reificación a aquellos procesos por los cuales la gente se trata a sí misma y a los demás como categorías abstractas, considerando así el 
bajo sobre el yo coherente con la dinámica capitalista y que maximiza la percepción acerca de la agencia individual orientada a la consecución de fines propios.

La discusión sobre si esta transformación devalúa la ontología de lo humano escapa a la empresa de este texto, pero lo que sin duda confirma en relación con los efectos de las redes sociales como medio y metáfora para concebir la identidad es su contribución al proceso de individualización contemporáneo, gracias a la insistencia de estas plataformas en la necesidad de diferenciación. Como han señalado algunos autores (Zajc, 2013; Rendueles, 2013a, 2013b), pese a la generalización de la denominación medios sociales, estos espacios refuerzan la concepción individual de la identidad social, potenciando una interpretación de la colaboración social desde la individualidad y los vínculos débiles, privilegiando de este modo las relaciones de bajo compromiso y puntuales, las relaciones líquidas planteadas por Bauman (2009). Rendueles (2013b) expresa como sigue esta tendencia a minusvalorar la idea de compromiso:

"Nos hemos acostumbrado a definirnos por nuestras preferencias: nuestros gustos musicales, nuestro estilo de vida, nuestras opiniones políticas... Nos concebimos como agregados de elecciones cuyo único fundamento es que han sido elegidas por nosotros. Desde esta perspectiva, cualquier forma de compromiso que no se pueda reducir a una elección inmediatamente reversible a voluntad, sólo puede ser entendida como un lastre [...] Internet ha acentuado esta autocomprensión hasta el paroxismo" (ibídem).

Traducido el sujeto en categorías conceptuales, y concebidas las relaciones con la alteridad de un modo crecientemente estratégico (Illouz, 2007) y autopromocional (Hearn, 2008; Marwick, 2013), tanto en sus relaciones fuertes como en las débiles, la cuantificación de los afectos, la medición de las preferencias y del reconocimiento de los otros (Honneth, 1998) son transformaciones que pueden entenderse como el siguiente paso coherente con este proceso de equiparación de lo humano con otras dimensiones de la existencia.

De este modo y apoyándose en gran medida en la capacidad performativa del lenguaje metafórico (Lakoff y Johnson, 2009) y en el poder comunicativo de la imagen -la preeminencia de la iconicidad positiva (el pulgar arriba del like en Facebook o el corazón en Instagram y en Twitter)-, la expresión de los afectos y afinidades en las redes sociales se ha potenciado desde estos espacios bajo una perspectiva cuantificadora, donde categorías cualitativas (los afectos, por ejemplo) se han traducido como cuantificables (cuántos likes, cuántos retuits).

El principal problema comunicativo de la cuantificación es que el sistema tiende a unificar afectos no comparables, es decir, uniformiza para poder establecer comparaciones con los otros. Aunque Facebook ha desarrollado la opción reacciones (figura 1), que amplía las posibilidades de expresar "empatía” en la plataforma (Alba, 2015), cualesquiera que fueran sus significados de partida, todo emoji acaba siendo reducido a un número final de interacciones que permite comparar

concepto como si fuera lo real, siendo tratado el sujeto no de acuerdo con sus cualidades humanas, sino como un medio para un fin, un objeto o una mercancía. 
el éxito social de la publicación -entendido el éxito como la capacidad para atraer la atención-con los ítems precedentes y con las publicaciones de otros usuarios de la plataforma.

Figura 1. Imagen de los nuevos emojis que ofrece la utilidad reacciones en Facebook. Fuente: De Sancha Rojo (2015).

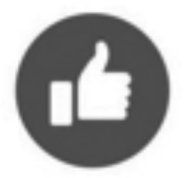

Me gusta

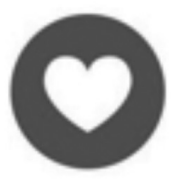

Me encanta

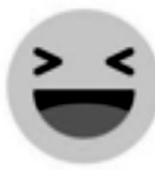

Me divierte

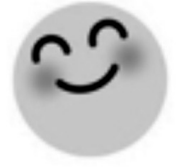

Me alegra

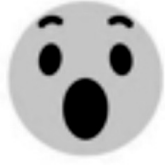

Me asombra

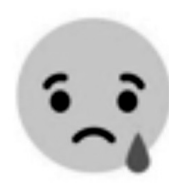

Me entristece

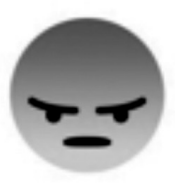

Me enfada

El significado de la opción favoritos en Twitter, por ejemplo, es aún más compleja en cuanto al número de significados que los usuarios le otorgan. Así, Gorrell y Bontcheva (2016) han analizado la amplitud de usos de los favoritos para los usuarios: como marcadores de tuits que quieren recuperar en el futuro pero no compartir, como forma de agradecer un tuit, expresar conformidad, cerrar una conversación, autopromocionarse, etc. Esta variedad en los significados atribuidos a dicha utilidad pudo comprobarse en 2015 con las protestas y comentarios irónicos de los usuarios a raíz de la transformación icónica realizada por la plataforma, pasando de ser representado con una estrella a un corazón (VVAA, 2015).

Pese a esta variedad semiótica, cuando un usuario ubica el ratón sobre un tuit y observa que ese ítem cuenta con diez favoritos, esta diversidad de significados y usos queda reducida a un dato numérico, que aporta una información especialmente útil para el lenguaje máquina y el desarrollo de rankings y clasificaciones de temas tendencia, pero que reduce radicalmente los significados construidos por los sujetos en sus interacciones.

Las aplicaciones y utilidades que permiten la cuantificación y medición de las acciones e interacciones del individuo no se limitan a medir de modo inocuo; muy al contrario, establecen puntos de comparación del sujeto consigo mismo y con los otros, al mismo tiempo que contribuyen a fijar qué es lo socialmente deseable, dónde se ubica el sujeto en relación con ese yo que se apunta como ideal y cómo deben concebirse los cuidados del yo en relación con estos medios.

\subsection{Un yo en competencia con los otros}

Dentro de la retórica neoliberal, el diseño de utilidades de cuantificación en las redes sociales -número de likes, retuits, favoritos, comentarios, etc.-, así como la posibilidad de conectar estas plataformas a otros servicios que extraen los datos generados por el usuario -Klout, PeerIndex, Favstar, Tweetreach ${ }^{5}$, etc.-, configura un sistema de recompensas de las ac-

Tweetreach es una herramienta que mide la visibilidad potencial que puede lograr cada publicación de un usuario. Este servicio toma los datos de la API de Twitter para ofrecer el dato de la audiencia potencial que podría haber visto uno de sus tuits mediante la suma de las audiencias personales de cada usuario que hizo retuit.

24 | n²4, pp. 13-36 | doxa.comunicación 
ciones de los usuarios en el que se favorece la competencia por la atención de las audiencias sociales, al mismo tiempo que se traslada la lógica de la acumulación capitalista al ámbito de las relaciones y afectos.

A ello contribuye la cuantificación y la visibilidad que dan las diferentes plataformas a los ítems que despiertan más reacciones en los demás. Twitter, por ejemplo, envía avisos por correo electrónico a modo de boletín de noticias en los que destaca las publicaciones que han despertado mayor número de interacciones entre los contactos del receptor, al mismo tiempo que ofrece al usuario la posibilidad de conocer el "alcance" de cada tuit propio e incluso promocionarlo (figura 2). Facebook, por su parte, combina los criterios de proximidad del vínculo, actualidad y capacidad de atraer la atención a la hora de presentar las noticias que configuran el muro de actualizaciones; al tiempo que potencia la nostalgia y la asimilación de Facebook a un álbum personal, con avisos que invitan al sujeto a recordar una publicaciones antiguas, como la utilidad "Un día como hoy". De este modo, estas aplicaciones y utilidades del interfaz tienen una considerable influencia en la construcción del consenso en torno a qué es lo importante, deseable, aceptable, digno de recordar, etc.

Figura 2. Captura de pantalla de la información que ofrece Twitter sobre el alcance en términos de audiencia de cada tuit y las posibilidades de promocionarlo.

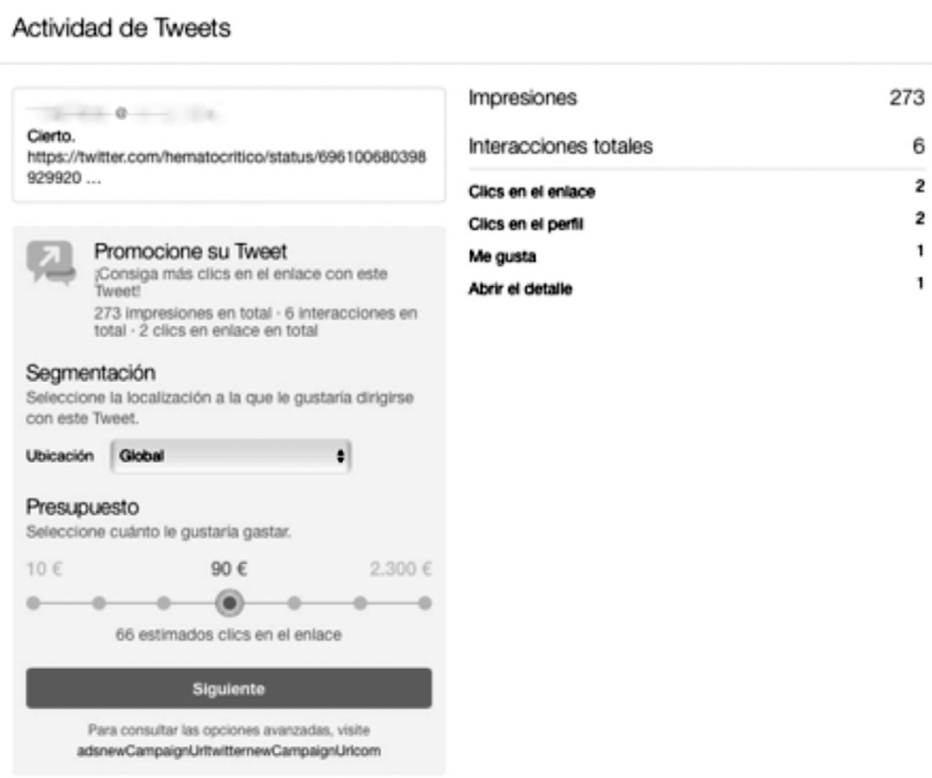

En su estudio sobre la presentación de la identidad para la búsqueda de pareja en webs de citas, Illouz (2007) revisó la influencia del capitalismo y del auge del psicologismo del siglo XX en el empleo de las emociones por parte de los usuarios. 
Según la autora, el capitalismo emocional ha producido una reinterpretación en torno al valor de uso de las emociones, que ahora tenderán a ser usadas estratégicamente por los individuos en sus relaciones. Por otra parte, Illouz considera que publicar el perfil online convierte al yo privado en un yo público, mostrándose el sujeto ante "una audiencia abstracta y anónima que, sin embargo, no es un público (en el sentido habermasiano del término) sino más bien un conjunto de yo [sic] privados" (ibídem: 170). Desde la perspectiva de este trabajo, se entiende como más adecuado el término audiencia, para referirse al tipo de relaciones entabladas en estos espacios, donde la búsqueda de visibilidad masiva guía muchas de las estrategias comunicativas.

En esta representación pública de la identidad ante una audiencia -frente a la idea de comunidad, por ejemplo-, los individuos tienden a adoptar fórmulas de representación propias de los medios de comunicación de masas y de la comunicación comercial: la postura corporal, la gestualidad, el retoque de la imagen, etc. La hiperritualización (Goffman, 1991) de las imágenes de perfil en las redes sociales puede afirmarse como una constante para garantizar que se traslada a las audiencias un conjunto de signos que serán capaces de interpretar en el sentido que esperan, comunicando así una personalidad y estilo reconocibles.

Otra de las consecuencias que señala Illouz es que el modo en que los sujetos deben amoldarse a estas herramientas y a los imaginarios que las atraviesan potencia un predominio de la “ideología liberal de la 'elección'” (Illouz, 2007: 171), una situación análoga a la del mercado en el que el usuario debe hacer la mejor elección posible. De este modo, si el sujeto se entiende a sí mismo y a los demás en una situación de libre mercado, se encuentra, por tanto, en competencia con otros, y en este marco deberá venderse para ser considerado más atractivo que ese otro indeterminado con el que compite, tal y como funciona la comunicación de cualquier marca comercial.

Desde esta perspectiva, la lucha por la atención se convierte en un bien escaso que los individuos en las redes sociales son animados a alcanzar mediante mecanismos de cuantificación que les permiten compararse con los otros. Si el consumo se ha erigido en lenguaje para la expresión de la identidad (Giddens, 1994; Verdú, 2007), en las redes sociales a esta información de consumo se añaden los datos en torno al volumen de vinculaciones e interacciones que el sujeto es capaz de acumular. Así, del mismo modo que "el estatus es un juego intrínsecamente competitivo" (Heath y Potter, 2009: 132) y lleva a los sujetos a emprender carreras de consumo con sus vecinos para mostrar su estatus vía consumo visible, en las redes sociales los sistemas que visibilizan las vinculaciones e interacciones potencian la percepción de hallarse en competencia por los bienes de estos espacios: atención y expresión de afectos, esencialmente.

Para lograr captar la atención, muchos usuarios desarrollan acciones dirigidas a atraer a las audiencias, empleando la imagen, el gif y el vídeo como reclamos más atractivos per se que el texto. Asimismo, los usuarios tienden a definir un posicionamiento que les diferencie de los otros, llegando incluso a incorporar a sus publicaciones etiquetas que son tendencia en la plataforma en la que se publica, aun cuando no exista relación alguna entre la temática de lo compartido y el 
hashtag. Ésta es una práctica a la que Abidin (2014) denomina "secuestro" (hijacking) ${ }^{6}$, dicha práctica busca acceder a una mayor visibilidad y difusión para las propias publicaciones, aún a riesgo de convertir el contenido propio en spam para los usuarios que utilizan las etiquetas como folksonomía (figura 3).

Figura 3. Captura de pantalla de una publicación en Instagram que recurre a etiquetas populares con independencia de su coherencia con lo publicado.
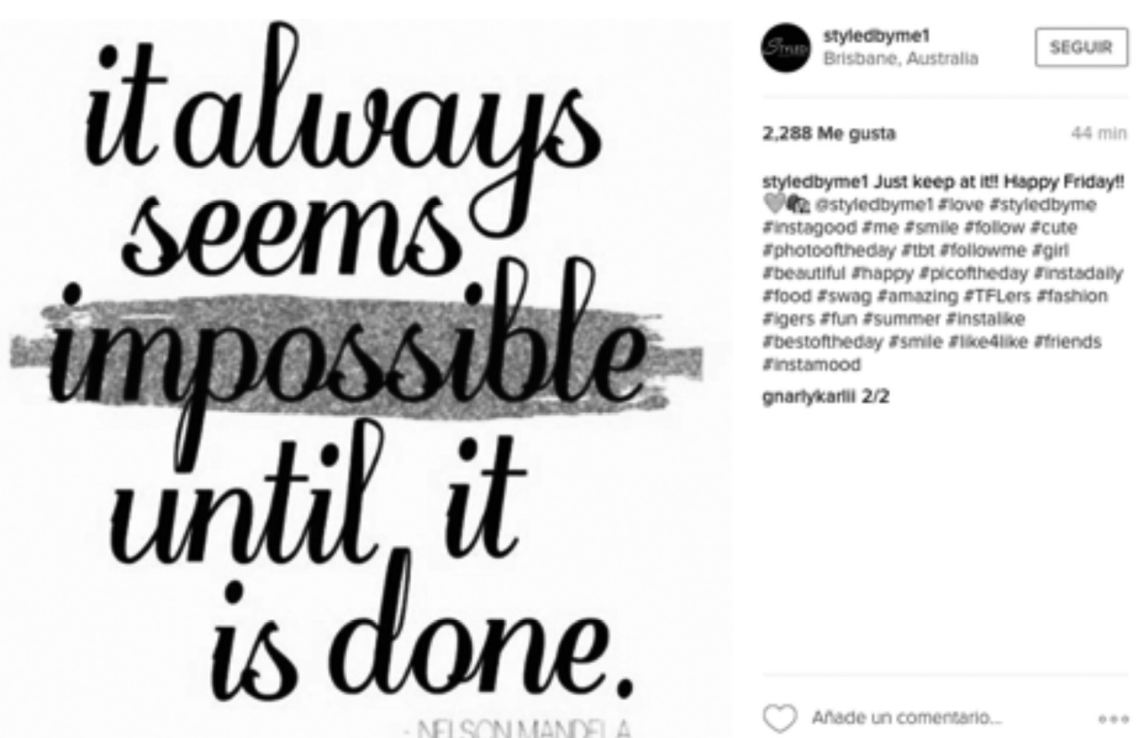

\subsection{La audiovisualización de la presentación del yo}

De acuerdo con Miller y Sinanan (2014), la generalización de la comunicación a través de la webcam estaría cambiando el modo en el que el sujeto se observa a sí mismo en la interacción comunicativa. Ello es debido a que estaría normalizando el hecho de verse mientras se está interactuando con los otros. En este sentido, también las redes sociales han contribuido en gran medida a que se haya vuelto común la posibilidad de construir una presentación visual y audiovisual de la identidad (Van der Molen, 2014: 65). De hecho, en el caso de los jóvenes, los usuarios que no publican fotografías de sí mismos en espacios como Facebook tienden a despertar sospechas entre los contactos de su red (Aziz, 2014; Caro Castaño, 2015).

6 En el ámbito informático, el hijacking hace referencia a toda técnica ilegal que lleve consigo el adueñarse o robar algo (generalmente información) por parte de un atacante, por lo que el término puede interpretarse en este contexto como el robo de la atención del usuario a través de un uso ventajista de las etiquetas que no contribuye en modo alguno a ordenar los contenidos generados por los usuarios en la red para que otros puedan encontrarlos, sino lograr visibilidad y autopromoción. 
Han ha llegado a plantear en esta línea la aparición de "la coacción icónica de convertirse en imagen” (2013: 31) dentro de la "sociedad de la transparencia", donde todo lo que no queda expuesto al régimen de lo visible queda automáticamente bajo sospecha. El sometimiento del cuerpo a la hipervisibilidad, para Han, constituye parte de su transformación en mercancía (ibídem: 51).

Sin embargo, frente a la promesa de espontaneidad y desvelamiento de lo auténtico de estos espacios, la comunicación mediada posibilita la repetición, el ensayo de la pose, el retoque en la pre y la postproducción en la presentación audiovisual del yo. De este modo, procesos propios de la producción y la realización audiovisual de los massmedia y sus claves estéticas de representación pasan a integrarse en prácticas comunicativas cotidianas y de cuidado del yo (Foucault, 1990). El trabajo sobre la representación audiovisual propia se convierte así en un ejercicio de autorrealización en un sentido casi literal: si la Segunda Modernidad trajo consigo el yo como tarea individual y diferencial, donde el sujeto debe exteriorizar su identidad para buscar la validación social (Giddens, 1994), las redes sociales se han convertido en la herramienta que permite la experimentación con esa construcción en la pantalla (Cover, 2014; Miller y Sinanan, 2014), en términos narrativos y audiovisuales.

Al mismo tiempo, ha enfrentado a los integrantes de la audiencia con la posibilidad de distribuir su propia imagen y discernir acerca de qué estrategias y tácticas contribuyen o no a la captación de la atención de los otros, introduciendo de este modo la lógica de la competencia y la acumulación. Los quince minutos de fama que planteó Warhol se convierten ahora en la búsqueda del retuit masivo, la consecución del mayor número de reacciones a la nueva foto de perfil o superar un determinado número de comentarios en cada nueva imagen o vídeo. Esta orientación finalista de la comunicación va condicionando los modos en que los usuarios se muestran textual y audiovisualmente.

Dado que no se trata sólo de cuidar el modo en el que se traduce el yo audiovisualmente, sino que también preocupa la respuesta que esta representación halla entre los usuarios, el sujeto desarrolla en este proceso de exteriorización una versión aspiracional de sí mismo. Como ha señalado Pérez-Chirinos (2012), en la narración que el sujeto hace de su devenir cotidiano en estos espacios

"se observa una mayor influencia de lo que uno aspira a ser (identidad cualitativa) que de lo que los demás esperan que sea (identidad social): se comparte lo especial, lo novedoso, lo que se disfruta, más que la confirmación de costumbres y rutinas (ibídem: 22)".

Construir y alimentar esa presentación aspiracional online requiere de una constante labor inmaterial por parte de los sujetos para la generación de contenidos propios. Así, como ha señalado Lasén (2012), es una práctica habitual entre los jóvenes la realización de "quedadas" de amigos para hacer sesiones fotográficas que posteriormente serán retocadas y publicadas en las redes sociales. Estas sesiones suelen hacerse en espacios no domésticos o, al menos, suelen salirse de los espacios que el sujeto normalmente muestra como su espacio cotidiano. Las imágenes producidas en estos encuentros 
suelen presentar un gran cuidado en la composición, el vestuario, maquillaje, etc. para hacer fotografías que típicamente replican la estética de los reportajes de revistas de moda, donde los retratados exploran esa pose más brillante que buscan que les represente, como señalaba Goffman (1991: 168), recurriendo al repertorio gestual hiperritualizado por los discursos de la moda, la publicidad y el cine.

Obviamente, la normalización de una presentación audiovisual estratégica del yo como forma de autorrealización y cuidado de uno mismo no surge con estos servicios web, aunque sean ellos los que más claramente han permitido el ejercicio de esta forma de concebir y expresar el yo hasta la fecha. La raíz cultural de este fenómeno es, al menos, doble. En primer lugar, debe señalarse la influencia de la cultura de la promoción, que asentó el valor competitivo y económico de la "semiosis artificial" creada por el marketing, la publicidad y las relaciones públicas en torno a la mercancía-signo (Wernick, 1994: 18). En segundo lugar, debe destacarse el peso de la cultura de los massmedia y las celebridades -entendidas éstas como versiones aspiracionales en las que se reconocen las audiencias (Marshall, 2011)-, cuyo ethos ha rodeado de un aura de glamour el hecho de ser visible y ha potenciado la concepción de la hipervisibilidad como una forma de poder contemporánea.

\section{Conclusiones}

En resumen, tras todo lo expuesto anteriormente y con todas las cautelas que exige la concepción de las redes sociales digitales como constructos sociotécnicos, se sostiene que estos espacios proponen una forma concreta de concebir la subjetividad y presentarse ante la alteridad que se ha denominado como identidad mosaico y que se caracteriza por:

a) potenciar una concepción distribuida de la identidad social, en continua co-creación y negociación de la representación de la posición del sujeto en la estructura de red, donde los contenidos que el usuario hace fluir por los vínculos siguen una lógica fragmentaria y de autoría diversa;

b) favorecer procesos de reificación -hacia el propio sujeto al normalizar el hecho de convertirse a sí mismo en objeto de consumo y hacia sus relaciones sociales- y de cuantificación del yo, mediante la lectura de las relaciones e interacciones de acuerdo con los sistemas de visibilización propuestos por cada plataforma (retuits, likes, favoritos, etc.);

c) promover una percepción del yo en competencia con los otros -por la atención, los afectos, el reconocimiento social, etc.-, una percepción enfatizada por los mecanismos de cuantificación de estos espacios que le plantean unidades de comparación; y

d) alentar la normalización de una presentación audiovisual estratégica del yo, donde la representación del cuerpo es concebida como una expresión de autenticidad y cercanía, aun cuando en esta presentación se tienda a adoptar la semiótica de los medios de comunicación de masas. 


\section{Bibliografía}

Abidin, C. (2014): “\#In\$tagLam: Instagram as a Repository of Taste, a Brimming Marketplace, a War of Eyeballs”, en Berry, M. y Schleser, M. (eds.): Mobile Media Making in an Age of Smartphones. New York: Palgrave Macmillan, pp. 119-128.

Alba, D. (2015): “Facebook Tests Emoji Reactions to Fix Its ‘Dislike’ Problem”, Wired.com, 08/10/2015. Disponible en: http://www.wired.com/2015/10/facebook-reaction-emoji [Consultado el 26/02/2016].

Andrejevic, M. (2010): “Social Network Exploitation”, en Papacharissi, Z. (ed.): A Networked Self: Identity, Community and Culture on Social Network Sites. New York: Routledge, pp. 82-101.

Arcila, C. (2011): “Análisis comparativo de la presentación personal virtual en diferentes espacios de interacción”, Fonseca Journal of Communication, n. 3, pp. 153-169. Disponible en: http://revistas.usal.es/index.php/21729077/article/view/11907/12271 [Consultado el 17/02/2016].

Arda, Z. (2011): Yo soy mi imagen 2.0: Aproximación a formas contemporáneas de construir la identidad humana [tesis doctoral inédita]. Castelló de la Plana: Universitat Jaume I. Disponible en: http://www.tesisenred.net/handle/10803/81855 [Consultado el 05/02/2016].

Ardèvol, E. y Gómez-Cruz, E. (2012): "Las tecnologías digitales en el proceso de investigación social: reflexiones teóricas y metodológicas desde la etnografía virtual”, en CIDOB (ed.): Políticas del conocimiento y dinámicas interculturales: acciones, innovaciones, transformaciones (pp. 187-204). Barcelona, CIDOB. Disponible en: http://goo.gl/WL3IIq [Consultado el 11/02/2016].

Aziz, F. (2014): “Transactions visuelles: Facebook, ressource de la rencontre amoureuse”, Études Photographiques, n. 31. Disponible en: http://etudesphotographiques.revues.org/3388 [Consultado el 22/01/2016].

Bauman, Z. (2009): Comunidad. En busca de seguridad en un mundo hostil. Madrid: Siglo XXI.

Belk, R. W. (1988): “Possessions and the extended self”, Journal of Consumer Research, vol. 15, pp. 139-168. Disponible en: http://goo.gl/jKH6ji [Consultado el 06-11-2015].

Bird, E. (2010): "From fan practice to mediated moments: The value of practice theory in the understanding of media audiences”, en Brauchler, B. y Postill, J. (eds.): Theorising media and practice. New York: Berghahn, pp. 85-104.

Blumer, H. (1980): "Mead and Blumer: The Convergent Methodological Perspectives of Social Behaviorism and Symbolic Interactionism”, American Sociological Review, vol. 45, n. 3, pp. 409-419.

Boyd, D. M. y Ellison, N. B. (2008): “Social Network Sites: Definition, History, and Scholarship”, en Journal of Computer-Mediated Communication, vol. 13, pp. 210-230. Disponible en: http://goo.gl/jTw2fh [Consultado el 28/02/2016].

Brown, W. (2003): “Neo-liberalism and the End of Liberal Democracy”, Theory y Event, vol. 7, artículo 1.

Del Fresno, M. (2012): El consumidor social. Reputación online y 'social media'. Barcelona: Editorial UOC. 
Cáceres, M. D., Ruiz, J. A. y Brändle, G. (2009): “Comunicación interpersonal y vida cotidiana. La presentación de la identidad de los jóvenes en Internet”, CIC: Cuadernos de Información y Comunicación, n. 14, pp. 213-231. Disponible en: http://eprints.ucm.es/22541/ [consultado el 12/01/2016].

Caro Castaño, L. (2012): “Identidad mosaico. La encarnación del yo en las redes sociales digitales”, Telos: Cuadernos de Comunicación e Innovación, n. 91, pp. 59-68. Disponible en: http://goo.gl/rRgJKE [Consultado el 26/01/2016].

- (2015): “Translated Bodies: The Hyper-Ritualization in the Representation of the Male Body among Spanish Youth in Social Networking Sites", en Rey, J. (ed.): The Male Body as Advertisement: Masculinities in the Hispanic Media. New York: Peter Lang, pp. 139-148.

Colombo, F. (2013): “Control, Identity, Self-Governmentality. A Foucauldian Approach to Web 2.0”, en Tomanić, I., Carpentier, N., Nieminen, H., Pruulmann-Venerfeldt, P., Kilborn, R., Sundin, E. y Olsson, T. (eds.): Past, Future and Change: Contemporary Analysis of Evolving Media Scapes. Ljubljana: Biblioteca Nacional y Universitaria, pp. 309-327. Disponible en: http:/ /www.researchingcommunication.eu/C23_book8.html [Consultado el 06/12/2015].

Couldry, N. (2008): "Mediatization or mediation? Alternative understandings of the emergent space of digital storytelling", New Media and Society, vol. 10, n. 3, pp. 373-391. DOI: 10.1177/1461444808089414

- (2014) “When Mediatization Hits the Ground”, en Hepp, A. y Krotz, F. (eds.): Mediatized Worlds: Culture and Society in a Media Age. Hampshire/New York: Palgrave Macmillan, pp. 54-71.

Cover, R. (2014): "Becoming and Belonging. Performativity, Subjectivity, and the Cultural Purposes of Social Networking”, en A. Poletti y J. Rak (Eds.): Identity Technologies. Madison: The University of Wisconsin Press, pp. 55-69.

Davis, J. (2012): “Prosuming Identity: The Production and Consumption of Transableism on Transabled.org”, American Behavioral Scientist, vol. 56, n. 4, pp. 596-617. DOI: 10.1177/0002764211429361

De Sancha Rojo, M. (2015): “Facebook estrena Reactions, una extensión que permite reacciones más allá del 'me gusta'”, HuffingtonPost.es, 08/10/2015. Disponible en: http://www.huffingtonpost.es/2015/10/08/facebook-reactionsemociones_n_8262308.html [Consultado el 05/03/2016]

Deresiewicz,W. (2011): “Generation Sell”, www.nytimes.com, 12/11/2011. Disponible en: http://goo.gl/6G9CJE [Consultado el 04/03/2016].

Deuze, M. (2012): Media Life. Cambridge/Malden: Polity Press.

Di Siena, D. (2011): “Ciudades de código abierto", Creatividad y Sociedad, n. 17, pp. 1-19. Disponible en: http://goo.gl/4TM4BR [Consultado el 07/03/2016].

Ellison, N. B., Steinfield, C. y Lampe, C. (2007): “The Benefits of Facebook 'Friends': Exploring the Relationship Between College Students' Use of Online Social Networks and Social Capital", Journal of Computer-Mediated Communication, vol. 12, n. 3. Disponible en: http://jcmc.indiana.edu/vol12/issue4/ellison.html [Consultado el 17/12/2015].

Foucault, M. (1990): Tecnologías del yo. Y otros textos afines. Barcelona: Paidós. 
Fuchs, C. y Sevignani, S. (2013): "What is Digital Labour? What is Digital Work? What's their Difference? And why do these Questions Matter for Understanding Social Media?”, tripleC, vol. 11, n. 2, pp. 237-293. Disponible en: http://www.triplec.at/index.php/tripleC/article/view/461 [Consultado el 09/02/2016].

Gergen, K. J. (2009): Relational Being: Beyond Self and Community. New York: Oxford University Press.

- (2010): El yo saturado. Dilemas de identidad en el mundo contemporáneo. Madrid: Paidós.

Giddens, A. (1994): Modernidad e identidad del yo. El yo y la sociedad en la época contemporánea. Barcelona: Península.

Goffman, E. (1967): Interaction Ritual: Essays in Face to Face Behavior. New York: Aldine Publishing Company.

- (1969): Where the Action Is. Three Essays. London: Allen Lane The Penguin Press.

- (1991): Los momentos y sus hombres. Textos seleccionados y presentados por Yves Winkin. Barcelona: Paidós.

- (2006): La presentación de la persona en la vida cotidiana. Buenos Aires: Amorrortu Editores.

Gorrell, G. y Bontcheva, K. (2016): “Classifying Twitter favorites: like, bookmark, or Thanks?”, Journal of the Association for Information Science and Technology, vol. 67, artículo 1, pp. 17-25. DOI: 10.1002/asi.23352

Hampton, K. N., Sessions, L., Rainie, L. y Purcell, K. (2011): Social Networking Sites and Our Lives. Washington D. C., Pew Internet y American Life Project. Disponible en: http://goo.gl/JRv3AS [Consultado el 04/03/2016].

Han, B. C. (2013): La sociedad de la transparencia. Barcelona: Herder.

Haraway, D. (2006): “A Cyborg Manifesto: Science, Technology, and Socialist- Feminism in the Late 20th Century”, en S. Stryker y S. Whittle (eds.): The Transgender Studies Reader. New York: Routledge, pp. 103-118.

Harper, R., Whitworth, E. y Page, R. (2012): “Fixity: Identity, Time and Durée on Facebook”, Selected Papers of Internet Research, vol. 2. Disponible en: http://spir.aoir.org/index.php/spir/article/view/8 [Consultado el 28-01-2016].

Hearn, A. (2008): “Meat, Mask, Burden: Probing the Contours of the Branded Self”, Journal of Consumer Culture, vol. 8, nº 2, pp. 197-217. DOI: $10.1177 / 1469540508090086$

Heath, J. y Potter, A. (2009): Rebelarse vende. El negocio de la contracultura. Madrid: Taurus.

Hine, C. (2004): Etnografía virtual. Barcelona: Editorial UOC.

Hjarvard, S. (2013): The mediatization of culture and society. Abingdon/New York: Routledge.

Honneth, A. (1998): “Entre Aristóteles y Kant. Esbozo de una moral del reconocimiento", Logos: anales del seminario de metafísica, n. 1, pp. 17-37. Disponible en: http://goo.gl/5t6t4e [Consultado el 18/02/2016].

Illouz, E. (2007): Intimidades congeladas. Las emociones en el capitalismo. Buenos Aires: Katz.

Jenkins, H. (2008): Convergence culture. La cultura de la convergencia de los medios de comunicación. Barcelona: Paidós. 
Jurgenson, N. (2012): “When Atoms Meet Bits: Social Media, the Mobile Web and Augmented Revolution”, Future Internet, vol. 4, n. 1, pp. 83-91. DOI: 10.3390/fi4010083

Kember, S. y Zylinska, J. (2012): Life after New Media. Londres: The MIT Press.

Kozinets, R. (2010): Netnography. Doing Etnographic Research Online. London: Sage.

Krotz, F. (2007): “The meta-process of mediatization as a conceptual frame”, Global Media and Communication, vol. 3, n. 3, pp. 256-260. DOI: 10.1177/17427665070030030103

Lakoff, G. y Johnson, M. (2009): Metáforas de la vida cotidiana. Madrid: Cátedra.

Lampel, J. y Bhalla, A. (2007): “The Role of Status Seeking in Online Communities: Giving the Gift of Experience”, Journal of Computer-Mediated Communication, vol. 12, n. 2. DOI: 10.1111/j.1083-6101.2007.00332.x

Lasén, A. (2009): “Tecnologías afectivas: de cómo los teléfonos móviles participan en la constitución de subjetividades e identidades", en Gatti, G., Martínez de Albéniz, I. y Tejerina, B. (eds.): Tecnología, cultura experta e identidad en la sociedad del conocimiento. Universidad del País Vasco, Bilbao, pp. 215-248.

- (2012): "Autofotos: subjetividades y medios sociales", en García-Canclini, N., Cruces, F. y Urteaga, M. (eds.): Jóvenes, culturas urbanas y redes digitales. Prácticas emergentes en las artes, el campo editorial y la música. Madrid: Fundación Telefónica, pp. 253-272.

Liu, H. (2007): "Social network profiles as taste performances", Journal of Computer-Mediated Communication, vol. 13, n. 1. DOI: $10.1111 /$ j.1083-6101.2007.00395.x

Martín Barbero, J. (2002): “Pistas para entre-ver medios y mediaciones”, Signo y Pensamiento, vol. 50, n. 41, pp. 13-20. Disponible en: http://www.redalyc.org/articulo.oa?id=86011596003 [Consultado el 16/02/2016].

- (2008): De los medios a las mediaciones. Comunicación, cultura y hegemonía. Barcelona: Gustavo Gili.

Marwick, A. E. (2013): Status Update: Celebrity, Publicity, and Branding in the Social Media Age. New Haven/London: Yale University Press.

Marwick, A. E. y Boyd, D. M. (2011): “To See and Be Seen: Celebrity Practice on Twitter”, Convergence: The International Journal of Research into New Media Technologies, vol. 17, n. 2, pp. 139-158. Disponible en: http://con.sagepub.com/content/17/2/139 [Consultado el 02/01/2017].

McLuhan, M. (1980): Comprender los medios de comunicación: las extensiones del ser humano. Barcelona: Paidós.

Mead, G. H. (2009): Mind, Self, and Society: From the Standpoint of a Social Behaviorist, (vol. 1). Chicago: University of Chicago Press.

Meyrowitz, J. (1985): No Sense of Place: The Impact of Electronic Media on Social Behavior. New York: Oxford University Press. 
Miller, D. y Sinanan, J. (2014): Webcam. Cambridge: John Wiley y Sons.

Miller, V. (2008): “New Media, Networking and Phatic Culture”, Convergence: The International Journal of Research into New Media Technologies, vol. 14, n. 4, pp. 387-400. DOI: 10.1177/1354856508094659

Mischel, W. y Morf, C. C. (2005): "The Self as a Psycho-Social Dynamic Processing System: A Meta-Perspective on a Century of the Self in Psychology”, en Leary, M. R. y Tangney, J. P. (eds.): Handbook of Self and Identity. New York: The Guilford Press, pp. 15-43.

Mitchell, W. J. (2007): “Ciudades inteligentes”, UOC Papers: revista sobre la sociedad del conocimiento, n. 5, pp. 3-9. Disponible en: http://www.uoc.edu/uocpapers/5/dt/esp/mitchell.html [Consultado el 05/01/2016].

Moles, A. (1978): Sociodinámica de la cultura. Buenos Aires: Paidós.

Myers, G. (2010): “Stance-taking and public discussion in blogs”, Critical Discourse Studies, vol. 7, n. 4, pp. 263-275. DOI: $10.1080 / 17405904.2010 .511832$

Nakamura, L. (2014): “Cyberrace”, En A. Poletti y J. Rak (eds.): Identity Technologies. Madison: The University of Wisconsin Press, pp. 42-54.

Page, R. (2012): “The linguistics of self-branding and micro-celebrity in Twitter: The role of hashtags", Discourse y Communication, vol. 6, n. 2, pp. 181-201. DOI: 10.1177/1750481312437441

Pérez-Chirinos, V. (2012): “Identidad y redes sociales: construcción narrativa del yo hipertextual”, en Austral Comunicación, vol. 1, no 1, pp. 9-25. Disponible en:http://www.austral.edu.ar/ojs/index.php/australcomunicacion/article/view/1 [Consultado el 03/02/2016].

Pérez Subías, M. (2012): “Identidad digital: Introducción”, en Telos: Cuadernos de Comunicación e Innovación, n. 91, pp. 5458. Disponible en: https://goo.gl/lcTBJr [Consultado el 28/02/2016].

Pinch, T. J. y Bijker, W. E. (1984): “The Social Construction of Facts and Artefacts: Or How the Sociology of Science and the Sociology of Technology Might Benefit Each Other”, Social Studies of Science, vol. 14, n. 3, pp. 399-441.

Reig, D. (2012): Socionomía ¿vas a perderte la revolución social? Barcelona: Deusto.

Rendueles, C. (2013a): Sociofobia. El cambio político en la era de la utopía radical. Madrid: Capitán Swing Libros.

- (2013b): "Santa Catalina, el PIB y los cuidados", Espejismosdigitales.wordpress.com, 11/06/2013. Disponible en: https://goo.gl/U71Ilf [Consultado el 04/03/2016].

Rodríguez, D. (2013): Memecracia. Los virales que nos gobiernan. Barcelona: Gestión 2000.

Rowan, J. (2012): “Marcas, sujetos-empresa y otras formas de vida contemporánea”, Revista Quimera, n. 338, pp. 48-53. 
Sampedro, V. F. (2004): "Identidades mediáticas e identificaciones mediatizadas: Visibilidad y reconocimiento identitario en los medios de comunicación", Revista CIDOB d'afers internacionals, n. 66-67, pp. 135-149. Disponible en: http://www.jstor.org/stable/40586099 [Consultado el 30/01/2016].

Senft, T. (2008): Camgirls: Celebrity y Community in the Age of Social Networks. New York: Peter Lang Publishing, Inc.

Serrano-Puche, J. (2013): “Vidas conectadas: tecnología digital, interacción social e identidad”, Historia y Comunicación Social, n. 18, especial noviembre, pp. 353-364. DOI: http://dx.doi.org/10.5209/rev_HICS.2013.v18.44249

Shifman, L. (2014): Memes in Digital Culture. Cambridge: The MIT Press.

Sibilia, P. (2008): La intimidad como espectáculo. Buenos Aires: Fondo de Cultura Económica.

Silverstone, R. (2002): "Complicity and collusion in the mediation of everyday life", New Literary History, vol. 33, n. 4, pp. 761-780. Disponible en: http://muse.jhu.edu/journals/nlh/summary/v033/33.4silverstone.html [Consultado el 19/01/2016].

Smith, S. y Watson, J. (2014): “Virtually Me: A Toolbox about Online Self-Presentation”, en Poletti, A. y Rak, J. (ed.): Identity Technologies. Constructing the Self Online. Madison: The University of Wisconsin Press, pp. 70-95.

Thompson, J. B. (2001): El escándalo político. Poder y visibilidad en la era de los medios de comunicación. Barcelona: Paidós.

- (2010): Los media y la modernidad: una teoría de los medios de comunicación. Barcelona: Paidós.

Van der Molen, J. B. (2014): Mediated Celebrity [tesis de máster]. Amsterdam: University of Amsterdam. Disponible en: http://dare.uva.nl/document/520058 [Consultado el 09/01/2016].

Van Dijck, J. (2013): “'You have one identity': performing the self on Facebook and LinkedIn”, en Media, Culture and Society, vol. 35, nº 2, pp. 199-215. DOI: 10.1177/0163443712468605

Verdú, V. (2007): Tú y yo, objetos de lujo. Barcelona: Random House Mondadori.

Vivienne, S. (2012): "Mediating identity narratives: a case study in queer digital storytelling as everyday activism”, en Fragoso, S. (ed.): AOIR selected papers of Internet research (IR 12.0): performance and participation (pp. 1-19). Disponible en: http://eprints.qut.edu.au/56941/ [Consultado el 01/02/2016].

VVAA (2014): "Allison Arling-Giorgi: For Gen Y, brands are our peers", Pbs.org, 18/02/2014. Disponible en: http://goo.gl/DLXFlo [Consultado el 04-03-2016].

VVAA (2015): “Twitter cambia las estrellas de favoritos por $\bullet$ corazones”, HuffingtonPost.es, 03/11/2015. Disponible en: http://www.huffingtonpost.es/2015/11/03/twitter-corazones_n_8461276.html [Consultado el 24/02/2016].

Walther, J. B. y Parks, M. R. (2002): "Cues Filtered Out, Cues Filtered In: Computer-Mediated Communication and Relationships”, en Knapp, M. L. y Daly, J. A. (eds.): Handbook of Interpersonal Communication. Thousand Oaks: Sage, pp. 529-563. 
Wernick, A. (1994): Promotional Culture. Advertising, Ideology, and Symbolic Expression. London: Sage.

Woermann, N. (2012): “On the Slope Is on the Screen: Prosumption, Social Media Practices, and Scopic Systema in the Freeskiing Subculture”, American Behavioral Scientist, vol. 56, n. 4, pp. 618-640. Disponible en: http://abs.sagepub.com/content/56/4/618.refs [Consultado el 02/01/2017].

Zajc, M. (2013): “Social media, prosumption, and dispositives: New mechanisms of the construction of subjectivity”, Journal of Consumer Culture [prepublicación en línea], 04-07-2013. DOI: 10.1177/1469540513493201

Zappavigna, M. (2012): Discourse of Twitter and Social Media: How We Use Language to Create Affiliation on the Web. London/New York: Bloomsbury Publishing.

Zhao S., Grasmuck S. y Martin J. (2008): “Identity construction on Facebook: digital empowerment in anchored relationships”, en Computers in Human Behavior, no 24, pp. 1816-1836. DOI:10.1016/j.chb.2008.02.012 